\title{
Disturbances in the voluntary recruitment order of anterior tibial motor units in bradykinesia of Parkinsonism ${ }^{1}$
}

\author{
LENNART GRIMBY AND JAN HANNERZ
}

From the Department of Neurology, Karolinska Sjukhuset, Stockholm

SUMMARY The recruitment of motor units is studied with an electromyographic technique for secure identification of single motor unit potentials. It has been shown in previous studies that the recruitment order is different in tonic and in phasic activities; in tonic activity the recruitment order is stable and low frequency units are always recruited before high frequency units; in phasic activity, however, the recruitment order is unstable and units with a higher frequency range may be recruited before units with lower frequency range. In this investigation the shifts between tonic and phasic recruitment order in voluntary contraction were compared in normal subjects and in patients with severe bradykinesia of Parkinsonism. Upon initiation of a voluntary contraction in a normal subject, phasic recruitment order may be used for a few $100 \mathrm{msec}$ but tonic recruitment order then takes over. In bradykinetic patients, however, this shift from phasic to tonic recruitment order is delayed. After termination of tonic voluntary contraction in a normal subject, phasic recruitment order can again be used after a few seconds. In bradykinetic patients, however, the shift back from tonic to phasic recruitment order is also delayed. In favourable experimental situations the shift from phasic to tonic recruitment order can be normalized by passive stretch of the muscle and the shift from tonic to phasic recruitment pattern by unloading the muscle. It is discussed whether the pathological recruitment in bradykinesia might be due to disturbed gamma loop function.

In animals different motor units of skeletal muscle exhibit differences in contraction time (Denny-Brown, 1929), in the tendency towards tonic discharge (Granit et al., 1956) and in fatiguability (Edström and Kugelberg, 1968).

The corresponding differences should exist in man. It is thus functionally essential that human motor units should be recruited in the order that is most adequate for the work intended. Electromyographic recordings can be arranged in such a way that it is possible to recognize single motor unit potentials with full certainty. The recruitment order of human motor units can thus be studied.

It has been shown in previous papers that the recruitment order of motor units is different in tonic and in phasic voluntary activity in normal man (Grimby and Hannerz, 1968) as well as in

1 This investigation was supported by grants from Karolinska Institutet, Stockholm. tonic and phasic exteroceptive reflex activity in 'spinal' man (Grimby and Hannerz, 1970). In tonic activity the motor units are recruited in a stable order. The motor units first recruited discharge at a low frequency and at regular intervals. In phasic activity, however, the recruitment order varies with the pre-existing state of facilitation in the motoneurone pool and with the properties of the eliciting stimulus (Hannerz and Grimby, 1973). The units first recruited may discharge in high-frequency bursts at irregular intervals (Grimby and Hannerz, 1973b).

The units of tonic and the units of phasic activity also have different discharge frequencies when they are active simultaneously in maximum voluntary contraction (Hannerz, 1973). It seems likely that each motor unit tends to discharge at its fusion frequency (Eccles et al., 1958) and that the low-frequency units of tonic activity have longer contraction times and the high-frequency 
units of phasic activity have shorter contraction times. The discharge frequency ranges (Hannerz, 1973) and the contraction times (Buchthal and Schmalbruch, 1969) reported for normal human muscles fit in well with this hypothesis. In patients with an upper motoneurone lesion, tonic spasticity causes excitatory bias for lowfrequency units and atrophy of type II muscle fibres (Edström et al., 1973) indicating a correlation between discharge frequency and contraction time even after supra-segmental lesions.

In animals slow-twitch units have a high resistance to fatigue, while fast-twitch units may be easily fatigued (Henneman and Olsson, 1965; Edström and Kugelberg, 1968; Kugelberg, 1973).

The tonic recruitment order must be optimal for sustained and precise work but inadequate for rapid movements, while the reverse must be true for the phasic recruitment order. There is now in progress a series of investigations with the aim of determining whether patients with different types of motor disturbances are able to select the recruitment order that is most adequate for the work intended (cf. also Grimby et al., 1973; Grimby and Hannerz, 1973c).

In this study we have shown that severely bradykinetic patients with Parkinsonism have a decreased ability to shift from phasic to tonic recruitment order when a sustained voluntary contraction is intended and to shift back from tonic to phasic recruitment order when instead a rapid voluntary contraction is intended. A preliminary report was given at the International EMG Congress in Brussels in September 1971 (Grimby and Hannerz, 1973a).

\section{METHODS}

The subjects in this study were 10 patients with Parkinsonism who had bradykinesia which was so severe that they were not able voluntarily to dorsiflex the foot promptly. Most of these patients were examined at several occasions at intervals up to several months. The studies were made before treatment with L-dopa or amantadine.

In the experiments the anterior tibial muscle was tested. The recording electrodes were placed proximally near to the tibia to exclude recordings from potentials set up from other muscles and comparatively near the muscle surface in order to avoid displacement of the recording electrodes. The motor unit potentials were recorded with one bipolar needle electrode (Disa 9013K0802) which has pre- viously been shown to have a selectivity neither too high nor too low for experiments of this type (Ashworth et al., 1967). For the recording conditions to be considered satisfactory it was a prerequisite that five different units should be readily identifiable. The potentials were amplified and displayed on two oscilloscopes-namely, a standard oscilloscope with continuous sweep and a Tektronix storage oscilloscope no. 564. The storage oscilloscope was triggered by the first positive deflection of the first potentials in each sequence, but its sweep was delayed $5 \mathrm{msec}$ by means of a delay unit so that the first potential also was completely displayed. All experiments were recorded on tape and the results obtained were confirmed by repeated playbacks and film recordings from the principal parts.

To be identified as deriving from a given unit a potential had to be of a characteristic size and shape and to remain unchanged duririg repeated activations. A second potential was defined as belonging to a second unit only if it were set up alternating with the first potential. A prerequisite for a third potential to be identified as deriving from a third unit was also that its size and shape excluded its being built upp of potentials number one and two. And so forth. BXod the criteria thus established it could be ascertaineo that the potentials evoked represented functionally separate units in the muscle. It is true that the possio bility cannot be excluded that some of the potentials may be derived from synchronized motor units, bu $\overrightarrow{0} \cdot \overrightarrow{0}$ this does not invalidate the conclusions drawn.

Mainly threshold contractions were studied, as the aim was to determine which unit was first recruited during the different contraction stage.

Histochemical analysis of the muscle area under study reveals a mixture of type I and II muscle fibres. Motor units are homogeneous as regards musclefibre type (Edström and Kugelberg, 1968). Two motor unit types are thus present in the muscle area studied.

\section{RESULTS}

As mentioned in the introduction, motor units can be recruited according to two different patterns both upon voluntary activation in normal subjects and upon reflex activation in patients with disturbed voluntary motoricity. In sustained activity one particular unit usually is the first recruited, irrespective of the mode of activation. This unit attains regular discharge $N$ frequencies well below $10 / \mathrm{sec}$ and has a maximal $\mathrm{N}$ frequency below $25 / \mathrm{sec}$. This low frequency unit is here called the B-unit to conform with previous papers. In twitch activity, however, the 


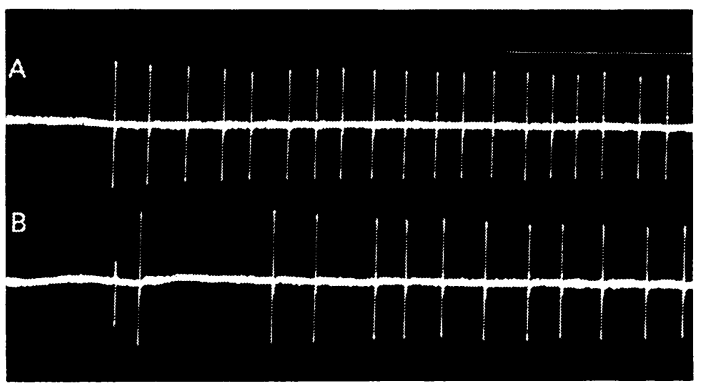

FIG. 1. Two initiations of tonic voluntary activity in a normal subject. (A) Upon subliminal voluntary facilitation of the motoneurone pool before the activation the 'B unit' has the lowest threshold from the very start. (B) Upon sudden initiation, however, the acticity starts with another unit but the ' $B$ unit' takes over within 100 msec. Time bar $1 \mathrm{sec}$.

recruitment order is unstable and some of the first recruited units do not attain regular discharge intervals until frequencies of $10-20 / \mathrm{sec}$ and these units have a maximum frequency above $30 / \mathrm{sec}$ even in sustained activity.

VOLLNTARY INITIATION OF SUSTAINED ACTIVITY IN NORMAL SUBJECTS A normal subject can always contract the anterior tibial muscle within a few hundred milliseconds.

If the subject facilitates the motoneurone pool subliminally a few seconds before the contraction. the activity is started directly by the B-unit (Fig. 1A).

If the subject makes a sudden contraction, however, the activity may start with another unit than the B-unit (Fig. 1B). In a threshold contraction the initiating unit normally only discharges once or twice and is always replaced by the Bunit after 100 to $200 \mathrm{msec}$ (Fig. 1B).

In a series of sudden contractions different units act as initiating unit. Some of these units discharge within a higher frequency range than the B-unit. In the experiment illustrated in Fig. 2 the initiating unit discharges only in bursts with relatively high frequency, while the B-unit is capable of regular low-frequency firing. The recruitment pattern in the initiation stage of sustained voluntary activity upon sudden contraction thus agrees with that in phasic activity.

VOLUNTARY INITIATION OF SUSTAINED ACTIVITY IN BRADYKINETIC PATIENTS The patients with Parkinsonism selected for this study usually could contract the anterior tibial muscle only with a considerable delay.

Upon cordinary voluntary effort the delay is of several seconds and the activity is initiated by the B-unit.

By extraordinary voluntary effort the patient may succeed in eliminating the delay. Then, however, the activity is initiated by other units while the B-unit remains delayed. In a threshold contraction the initiating units may dominate for several seconds before the B-unit takes over (Figs 3 and 4 ) in contrast with what is the case normally (Fig. 1).

The initiating units have no stable mutual recruitment order. In Fig. 3 they alternate irregularly and it cannot be anticipated which unit will discharge until the repetitive B-unit activity is finally established. Some of the initiating units discharge at a higher frequency than the B-unit. The initiating unit in the experiment illustrated in Fig. 4 discharges repeatedly only at frequencies above $10 / \mathrm{sec}$ and is either inactive or discharges just once when the drive is not sufficient for this frequency. The B-unit, however, discharges repeatedly even at frequencies as low as 6/sec.

The recruitment pattern of the initiation stage upon strong effort in bradykinetic patients thus agrees with that upon sudden contraction in normal subjects and with that in phasic activity.

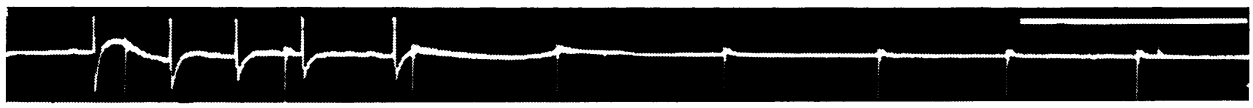

FIG. 2. Initiation of tonic voluntary activity in a normal subject. The initiating unit also discharges at a higher frequency than the B-unit when the two units are actice simultaneously.

Time bar $200 \mathrm{msec}$. 


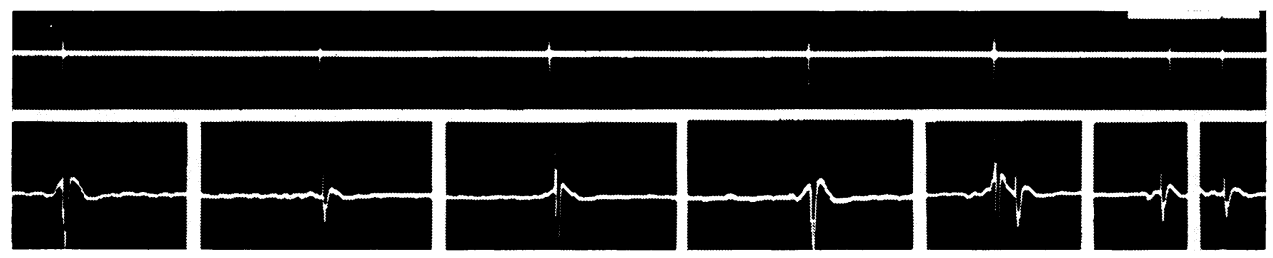

FIG. 3. Initiation of tonic voluntary activity in a bradykinetic patient. Three different units alternate as the unit of lowest threshold for several seconds before the B unit becomes dominant. Upper trace at low speed to show the continuous course (time bar I sec). Lower trace at a speed 50 times higher to show the characteristic shape of the potentials.

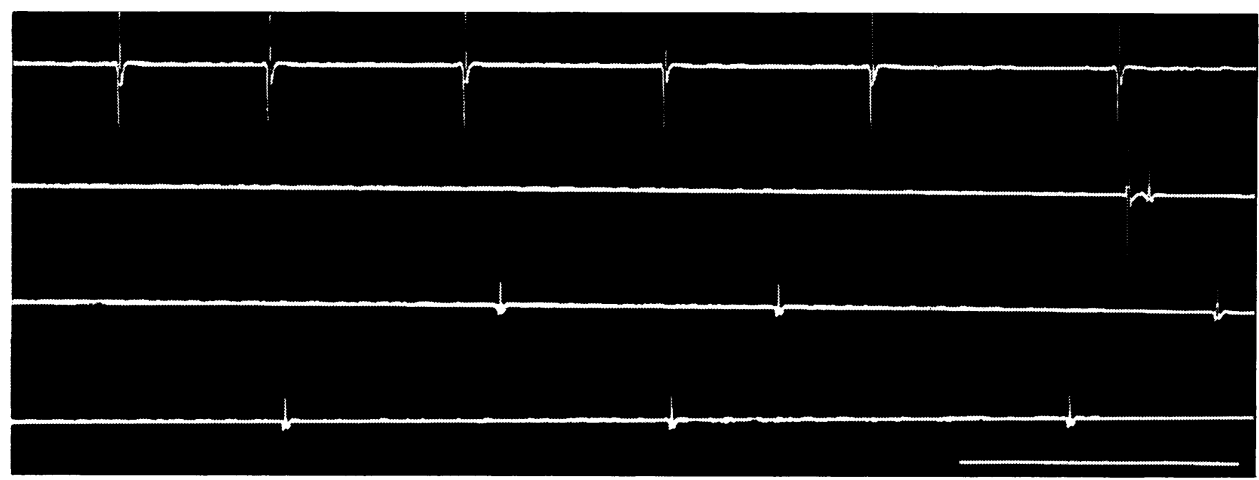

FIG. 4. Initiation of tonic voluntary activity in a bradykinetic patient. Each trace is a direct continuation of the trace above. The activity is initiated by another unit than the $B$ unit and it takes about a second before the $B$ unit becomes dominant. The initiating unit discharges repetitively only at frequencies above $10 / \mathrm{sec}$ and ceases when the drive is not sufficient for this frequency. The B unit, however, is capable of repetitive firing at frequencies down to $5 /$ sec. Time bar 100 msec.
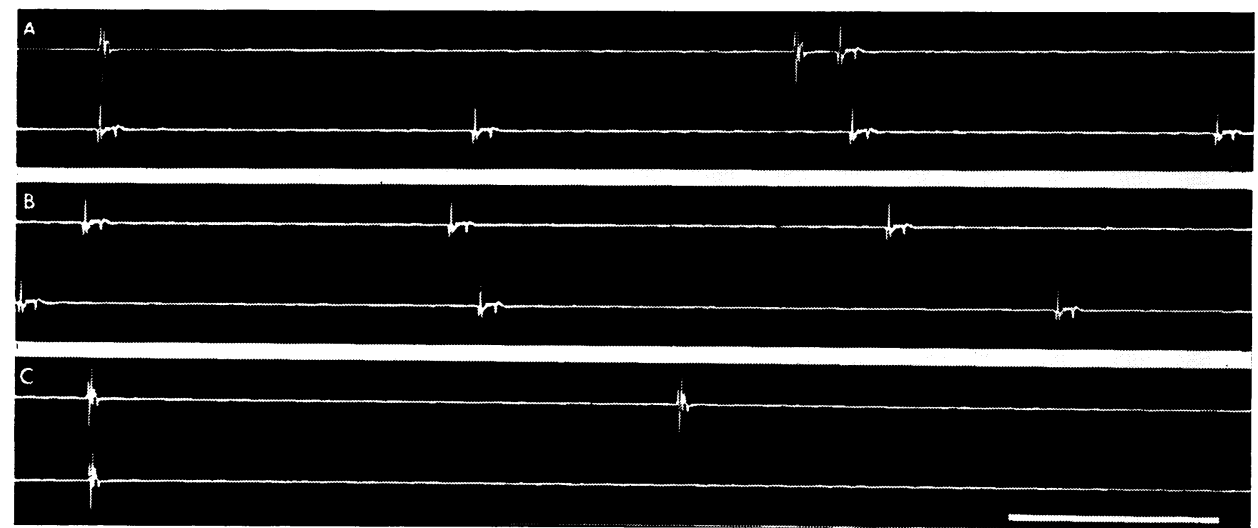

FIG. 5. Three initiations of tonic voluntary activity in a moderately bradykinetic patient. (A) Without interference from the examiner. (B) With passive stretch of the muscle. (C) With passive unloading of the muscle. In cach recording the lower trace is a direct continuation of the upper trace. Time bar 100 msec. 
The difference between the bradykinetic and the normal initiation stage is the much longer duration of the pathological initiation stage.

On comparison with the normal contraction in Fig. 1 the Parkinsonism contraction in Fig. 3 looks like a series of phasic initiations without tonic continuation. In the normal contraction in Fig. 2 and the Parkinsonism contraction in Fig. 4 the behaviour of the initiating unit is very similar, the main difference being the latency of the B-unit.

VOLUNTARY TERMINATION OF SUSTAINED ACTIVITY A normal subject can stop voluntary activity promptly. Our subjects with Parkinsonism, however, have difficulty in stopping repetitive B-unit activity when they have finally succeeded in establishing it.

The longer the B-unit has been active, the greater is the difficulty in stopping it. The period of voluntary control of the B-unit varies considerably from subject to subject, but it also varies to some degree from contraction to contraction in the same subject. Sometimes involuntary B-unit activity appears only after many minutes of a voluntary driving, sometimes it is doubtful whether the B-unit activity ever deserves to be called voluntary.

The involuntary B-unit activity disappears upon rest. The disappearance is accelerated if the subject contracts the antagonist muscles but delayed if the subject activates other muscles in the body-for example, makes a Jendrassic manoeuvre or if the subject is mentally stressed. The duration of the involuntary activity varies from a few seconds to several minutes.

Just after the involuntary B-unit activity has ceased the bradykinetic subject is able promptly to initiate B-unit activity voluntarily. A minute may pass before the initiation difficulties described above appear again. During this minute, however, the B-unit is first recruited irrespective of the mode of activation-that is, also when a rapid movement is intended.

Thus, instead of the inability to use tonic recruitment order the subject with Parkinsonism has, just after tonic contraction, a temporarily decreased ability to use phasic recruitment order.

There is no absolute correlation between the difficulty of a patient with Parkinsonism in changing from phasic to tonic recruitment order upon initiation of voluntary activity and his difficulty in changing from tonic to phasic recruitment order after termination of sustained voluntary activity. Severely bradykinetic subjects have both difficulties. However, subjects with Parkinsonism with slight bradykinesia but pronounced rigidity mainly have the difficulty in stopping tonic recruitment order.

INFLUENCE OF PASSIVE STRETCH OF MUSCLE ON RECRUITMENT ORDER IN VOLUNTARY INITIATION Normally, passive stretch of the anterior tibial muscle does not elicit any reflex activity. This procedure can, however, change the recruitment order upon voluntary initiation (Grimby and Hannerz, 1968).

In patients with Parkinsonism, passive stretch of the anterior tibial muscle may elicit repetitive B-unit activity in agreement with previous findings (for example, Hufschmidt, 1961). The degree of stretch can, however, be adjusted so that the effect on the motoneurone pool is subliminal. In subjects with moderately severe bradykinesia such a passive stretch may normalize the recruitment order upon voluntary initiation so that the B-unit is activated promptly and no initiation stage built up by other units appears, as is shown in Fig. 5. In subjects, with very severe bradykinesia, however, passive stretch has no significant effect on the voluntary recruitment order.

Normally, the effect on the recruitment order in voluntary initiation is obtained only by dynamic stretch and the original recruitment order is rapidly restored upon static stretch. In subjects with Parkinsonism, however, the changed recruitment order may be maintained for several seconds by merely static stretch, so that the angle in the foot joint decides the recruitment order, as shown in Fig. 5.

INFLUENCE OF UNLOADING OF MUSCLE ON RECRUITMENT ORDER IN VOLUNTARY INITIATION In a normal subject a voluntary B-unit activity pauses upon unloading of the muscle. If the normal subject makes a compensatory increase of its voluntary drive, another unit may discharge instead of the B-unit during this pause (Grimby and Hannerz, 1968).

In severely bradykinetic subjects, unloading of the muscle, and accompanying stretch of its 
antagonists, increases the delay of repetitive B-unit activity and thus also the role of the initiating units upon voluntary initiation, as shown in Fig. 5.

INFLUENCE OF PASSIVE STRETCH OF MUSCLE ON TERMINATION OF VOLUNTARY INITIATED ACTIVITY If the muscle is passively stretched when the Parkinsonism patient is told to terminate the contraction, the tendency towards subsequent involuntary activity increases.

INFLUENCE OF UNLOADING OF MUSCLE ON TERMINATION OF VOLUNTARILY INITIATED ACTIVITY Upon unloading of the active muscle, and accompanying stretch of its antagonists, the involuntary B-unit activity sometimes decreases, sometimes remains unchanged and sometimes increases.

A regular effect is, however, obtained in a specific experimental situation: when a passive stretch causing subliminal proprioceptive facilitation of the motoneurone pool is used to enforce voluntary effort, the Parkinsonism patient may be unable to stop the resulting B-unit activity voluntarily, while the experimenter can always stop it by stopping the passive stretch. In favourable experimental situations, such as that obtaining in the experiment illustrated in Fig. 5, it is possible to normalize the pathological initiation of voluntary activity in Parkinsonism by passive stretch and to normalize the pathological termination of voluntary activity by unloading the muscle.

\section{DISCUSSION}

The basis for this investigation is the difference in recruitment order between tonic and phasic voluntary activity in normal man. In tonic activity the recruitment order is stable and the first-recruited units discharge only at low frequencies. In phasic activity, on the other hand, the recruitment order varies with the pre-existing state of facilitation in the motoneurone pool and with the properties of the eliciting impulse and the first-recruited units may attain high discharge frequencies.

Before discussing the disturbances in voluntary recruitment order in bradykinesia of Parkinsonism, it must be stressed that they are found only in patients with subtotal akinesia before L-dopa treatment. It is, however, likely that disturbances also exist in less severe bradykinesia but that they cannot be discovered with the techniques used. Moreover, only the anterior tibial muscle is tested, as displacements of the recording electrodes can be avoided only in this muscle. Since, however, all muscles are influenced in Parkinsonism the results should be generally valid.

A normal subject can voluntarily initiate an activity with tonic recruitment order promptly. A severely bradykinetic subject, however, can make such an initiation only after considerable delay. Strong voluntary effort does not eliminate this delay but may result in activity with phasic recruitment order before the delayed activity with tonic recruitment order. Thus a bradykinetic patient is unable to initiate the mode of facilitation that is normally used for tonic activity promptly while, on maximum effort, he can stil use a mode of facilitation that is normally used only for phasic voluntary activity.

The disturbance in the recruitment order upor voluntary initiation increases upon passive stretch of the antagonist muscles. Inhibition from a rigid antagonist muscle may play a role in the inability of bradykinetic patients to star the normal mode of facilitation.

When a Parkinsonism patient has finally succeeded in initiating activity with tonic recruitment order, he has no difficulty in keeping it. On the contrary, there is a risk that he will lose cerebral control over the activity so that it continues involuntarily. He is now also unable to shift to phasic recruitment order in a normal way. It seems as though the path of facilitation that is used for tonic voluntary activity is both difficult to start and difficult to stop.

The role of the gamma loop in Parkinsonism is under debate. There are theories postulating overactivity (Rushworth, 1960) as well as theories postulating underactivity (Steg, 1964). The gamma loop is presumed to be one of those paths of facilitation of the motoneurone pool which gives rise to tonic recruitment order (Grimby and Hannerz, 1973a). It is thus tempting to think that it is the inflow from the muscle spindles that is difficult to start and difficult to stop voluntarily in bradykinesia.

Under favourable experimental conditions, 
the pathological recruitment order upon initiation of voluntary activity is normalized by passive stretch of the muscle and the pathological recruitment order upon termination of voluntary activity by unloading the muscle. In the experiment illustrated in Fig. 5 the recruitment order upon voluntary initiation is entirely dependent on the position of the extremity. These findings agree with the suggestion of decreased adaptability of muscle-spindle sensibility. However, none of our results proves that the afferent inflow is in fact abnormal.

Hagbarth et al. (1970) have studied the activity in muscle afferents in normal man and in Parkinsonism patients. Their results indicate that the inflow from the muscle-spindles at rest is more pronounced in subjects with Parkinsonism than in normal man, while the enhancement during voluntary effort is if anything smaller in Parkinsonism than normally. They could not, however, decide what role the disturbed gamma motoneurone innervation has in comparison with disturbed direct alpha motoneurone innervation.

Low-frequency motor units should have type I muscle fibres and high-frequency motor units type II muscle fibres (Edström et al., 1973). There are, however, intermediate forms between low- and high-frequency motor units (Hannerz, 1973) which have no equivalents in the muscle fibre classification.

Our finding that bradykinetic patients tend to use units with higher discharge frequencies instead of low-frequency units on initiation of sustained voluntary activity indicates that the relative role of type I muscle fibres may be decreased and that of type II muscle fibres increased in the very commencement of voluntary activity.

Our finding that bradykinetic patients during a period after sustained voluntary activity use lowfrequency units irrespective of the type of movement intended, indicates that type I muscle fibres are used more and type II muscle fibres less than normally in later contraction stages.

Histochemical studies of muscle biopsies from patients with Parkinsonism show that type II muscle fibres are atrophic, as after disuse, while type I muscle fibres are if anything hypertrophic, as after increased use (Edström, 1970).

The total effect of the starting and the stop- ping difficulties of low-frequency units seems to be increased or unchanged usage. The total effect of the opposite tendencies for highfrequency units seems instead to be decreased usage. It is in fact to be expected that the initiation stage is less important than the later contraction stage for the total usage of different unit types.

\section{REFERENCES}

Ashworth, B., Grimby, L., and Kugelberg, E. (1967). Comparison of voluntary and reflex activation of motor units. Functional organization of motor neurones. Journal of Neurology, Neurosurgery, and Psychiatry, 30, 91-98.

Buchthal, F., and Schmalbruch, H. (1970). Contraction times and fibre types in intact human muscle. Acta Physiologica Scandinavica, 79, 435-452.

Denny-Brown, D. (1929). The histological features of striped muscle in relation to its functional activity. Proceedings of the Royal Society Bulletin, 104, 371-411.

Eccles, J. C., Eccles, R. M., and Lundberg, A. (1958). The action potentials of the alpha motoneurones supplying fast and slow muscles. Journal of Physiology, 142, 275-291.

Edström, L. (1970). Selective changes in the sizes of red and white muscle fibres in upper motor lesions and Parkinsonism. Journal of Neurological Sciences, 11, 537-550.

Edström, L., and Kugelberg, E. (1968). Histochemical composition, distribution of fibres and fatiguability of single motor units. Journal of Neurology, Neurosurgery, and Psychiatry, 31, 424-433.

Edström, L., Grimby, L., and Hannerz, J. (1973). Correlation between recruitment order of motor units and muscle atrophy pattern in central motoneurone lesion: significance of spasticity. Experientia, 29, 560-561.

Granit, R., Phillips, C. G., Skoglund, S., and Steg, G. (1957). Differentiation of tonic from phasic alpha ventral horn cells by stretch, pinna and crossed extensor reflexes. Journal of Neurophysiology, 20, 470-481.

Grimby, L., and Hannerz, J. (1968). Recruitment order of motor units on voluntary contraction: changes induced by proprioceptive afferent activity. Journal of Neurology, Neurosurgery, and Psychiatry, 31, 565-573.

Grimby, L., and Hannerz, J. (1970). Differences in recruitment order of motor units in phasic and tonic flexion reflex in 'spinal man'. Journal of Neurology, Neurosurgery, and Psychiatry, 33, 562-570.

Grimby, L., and Hannerz, J. (1973a). Tonic and phasic recruitment order of motor units in man under normal and pathological conditions. New Developments in Electromyography and Clinical Neurophysiology, vol. 3, pp. 225233. Edited by J. E. Desmedt. Karger: Basel.

Grimby, L., and Hannerz, J. (1973b). Differences in recruitment order of motor units between early and late flexion reflex components in man. Acta Physiologica Scandinavica (In press.)

Grimby, L., and Hannerz, J. (1973c). Disturbances in the voluntary recruitment order of motor units in ataxia. (In preparation.)

Grimby, L., Hannerz, J., and Rånlund, T. (1973). Disturbances in the voluntary recruitment order of anterior tibial motor units in spastic parapareses upon fatigue. Journal of Neurology, Neurosurgery, and Psychiatry. (In press.)

Hagbarth, K.-E., Hongell, A., and Wallin, G. (1970). Parkinson's disease: afferent muscle nerve activity in rigid patients. Acta Societatis Medicorum Upsaliensis, 75, 70-76. 
Hannerz, J. (1973). Discharge properties of motor units in man. Experientia, 29, 45-46.

Hannerz, J., and Grimby, L. (1973). Recruitment order of motor units in man: significance of pre-existing state of facilitation. Journal of Neurology, Neurosurgery, and Psychiatry, 36, 275-281.

Henneman, E., and Olson, C. B. (1965). Relations between structure and function in the design of skeletal muscles. Journal of Neurcphysiology, 28, 581-598.

Hufschmidt, H.-J. (1961). Bausteine motorischer Regelung.
Schweizer Archiv für Neurologie Neurochirurgie, und Psychiatrie, 87, 260-280.

Kugelberg, E. (1973). Properties of the rat hind-limb motor units. New Developments in Electromyography and Clinical Neurophysiology, vol. 1, pp. 2-13. Edited by J. E. Desmedt. Karger: Basel.

Rushworth, G. (1960). Spasticity and rigidity: an experimental study and review. Journal of Neurology, Neurosurgery, and Psychiatry, 23, 99-118.

Steg, G. (1964). Efferent muscle innervation and rigidity. Acta Physiologica Scandinavica, 61, suppl. 225. 